\title{
Reasoning-Based Patient Classification for Enhanced Medical Image Annotation
}

\author{
Sonja Zillner \\ Corporate Technology, Siemens AG \\ Munich, Germany \\ sonja.zillner@siemens.com
}

\begin{abstract}
Medical imaging plays an important role in today's clinical daily tasks, such as patient screening, diagnosis, treatment planning and follow up. But still a generic and flexible image understanding is missing. Although, there exist several approaches for semantic image annotation, those approaches do not make use of practical clinical knowledge, such as best practice solutions or clinical guidelines. We introduce a knowledge engineering approach aiming for reasoning-based enhancement of medical images annotation by integrating practical clinical knowledge. We will exemplify the reasoning steps of the methodology along a use case for automatic lymphoma patient staging.
\end{abstract}

\section{Introduction}

The vision of THESEUS MEDICO 1 is to automatically extract the meaning from the medical images and to seamlessly integrate the extracted knowledge into medical processes, such as clinical decision making. In other words, the computer shall learn to find, catalogue and interpret medical images. This requires the semantic representation of medical images' content and the preprocessing of semantic image annotations for seamless integration into clinical applications.

The integration of practical clinical knowledge resources is an essential requirement to achieve the goals of MEDICO. Thus, we are aiming to enhance medical image annotations by integrating clinical knowledge, such as lymphoma staging systems. There exist several approaches for semantic image annotation, such as automatic image parsing [1], manual image annotation [2], 3], the extraction of information from DICOM headers and DICOM structured reports [4, or the automated extraction from radiology reports. Although those approaches provide the very important basis for semantic image annotation, they do yet not make use of practical clinical knowledge, such as best practice solutions or clinical guidelines for fine-tuning and customizing the established annotations to reflect the particular requirements of a clinical application or work flow.

Within the MEDICO project, one of the selected use case scenarios aims for improved image search in the context of patients suffering of lymphoma in

\footnotetext{
${ }^{1}$ http://theseus-programm.de/en-us/theseus-application-scenarios/medico/ default.aspx
} 
the neck area. Lymphoma, a type of cancer originating in lymphocytes, is a systematic disease with manifestations in multiple organs. The selected and discussed use case scenario is focusing on the automatic classification of lymphoma patients.

The contribution of this paper is to introduce a knowledge engineering approach aiming for enhanced medical image annotations by integrating clinical knowledge. The knowledge engineering steps will be exemplified along the selected use case. The Ann-Arbor Staging System of Hodgkin-lymphoma [5] provided us guidance in formally describing the knowledge base enabling the automatic inference of lymphoma patient staging results. Our aim for the knowledge base design was to establish an automatic staging system that maps each patient to uniquely one staging degree. We, thus, can automatically generate additional patient annotation data, that can be used for supporting the clinicians in their daily tasks. By integrating the patient's staging information, into other clinical applications, for instance, the quality of medical care can be optimized or the search and comparison of patients be improved. We have developed an OWL DL ontology that represents the Ann Arbor staging system together with lymphoma patient records and that is suitable for performing automatic lymphoma patient classification.

The remainder of the paper is organized as follows. Section 2 gives an overview of the KEMM Knowledge Engineering Methodology for the medical domain. In Section 3 we will introduce the knowledge resources the use case of ontologybased lymphoma patient staging is based on. Section 4 details the knowledge engineering steps exemplified along the selected use case scenario. Section 5] illustrates the benefits of the reasoning results by introducing a concrete example patient case and Section 6 discusses related approaches. Section 7 concludes this paper with an outlook on future work.

\section{Knowledge Engineering in the Medical Domain}

Given a formal and explicit representation of practical clinical knowledge, implicit conclusion can be derived. We conceive of reasoning-based enhancement of the ontology and the semantic annotations as an operation that is based on several knowledge resources - such as the information captured in the medical images, text-mining results on the basis of radiology reports, (fragments of) medical ontologies, and formal representations of clinical guidelines - and yields an outcome that can be directly used in clinical applications. A specific knowledge engineering methodology for the medical domain (KEMM) 6] has been designed. The knowledge engineering approach encompasses five steps, i.e. Knowledge Requirements Analysis, Requirements Decomposition, Knowledge Integration, Complex Query Composition, and Evaluation, and was derived from the experiences gained during the realization of a clinical use case within the MEDICO project. By specifying the knowledge engineering steps required for the integration of practical clinical knowledge into medical application, the KEMM module "Reasoning-Based Ontology Enhancement" is implemented. 


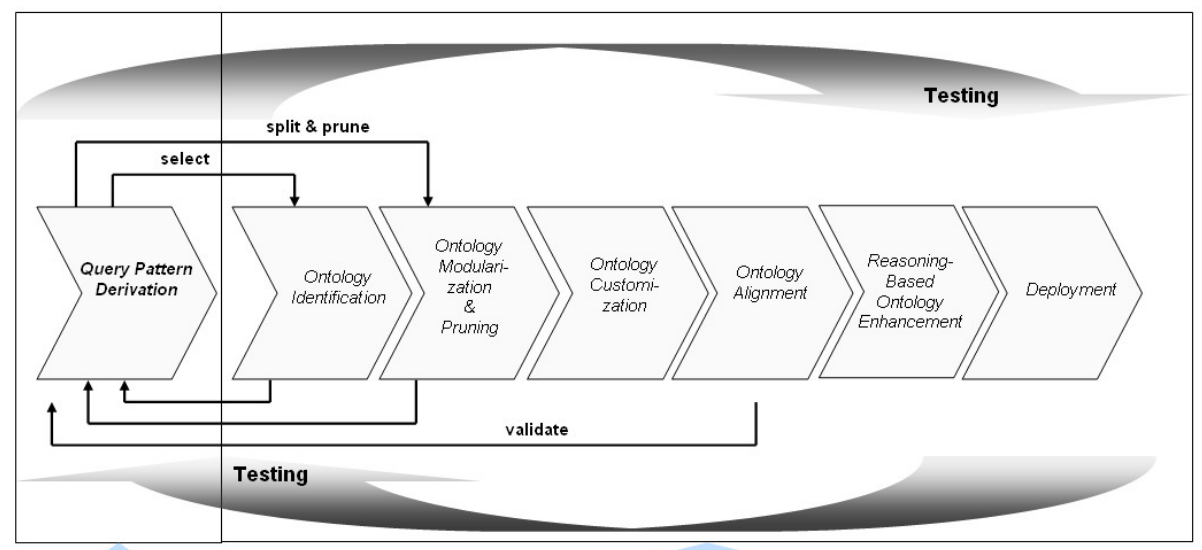

Fig. 1. The KEMM medical knowledge engineering methodology

\section{Knowledge Resources}

The approach for the automated staging application relies on three different knowledge resources that are described in the following.

Practical Clinical Knowledge. Only practical clinical knowledge that is explicitly and formally described can be integrated into other clinical applications. The Ann-Arbor Staging System for Hodgkin Lymphoma 5] documents precisely the classification of lymphoma patients. The staging depends on two criteria. The first criterion is the place where the malignant tissue is located, its type as well as its frequency of occurrence. The location can be identified with located biopsy as well as with medical imaging methods. The second criterion are systemic symptoms, such as night sweats, weight loss of more than 10 percent or fevers, caused by the lymphoma. Those systemic symptoms are called "B symptoms".

The principal stage is determined by the location of the tumor and reflects the grade of expansion of lymphoma occurrences. Four different stages are recognized:

- Stage I indicates that the cancer is located in a single region, either an affected lymph node or organ within the lymphatic system.

- In Stage II the cancer is located in two separated regions, an affected lymph node or an affected organ within the lymphatic system and a second affected lymph node area. Moreover, the affected areas are confined to one side of the diaphragm - that is, both are above the diaphragm or, both are below the diaphragm. 


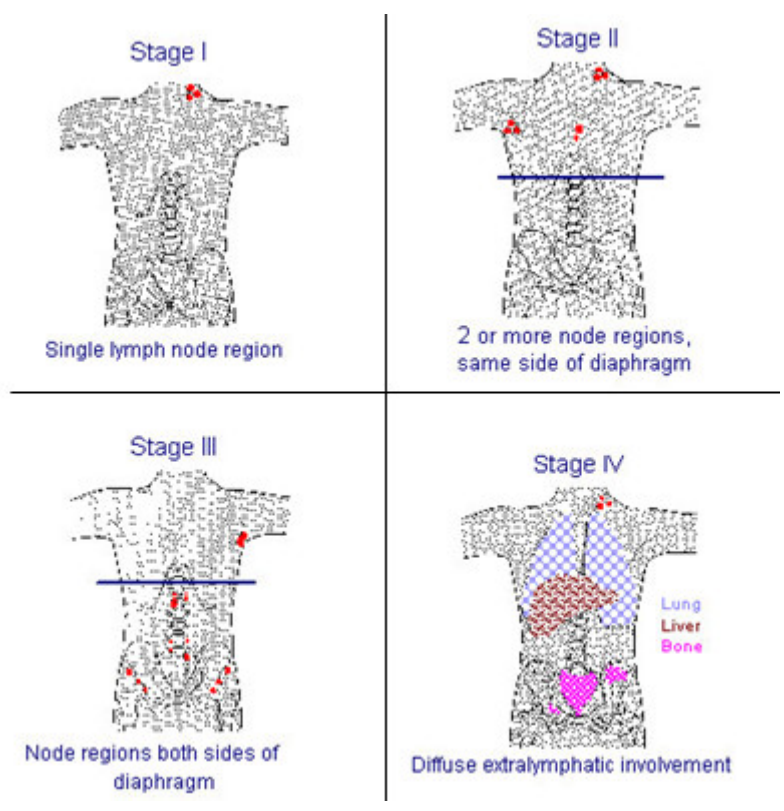

Fig. 2. Ann-Arbor Staging System (Source: http://training.seer.cancer.gov)

- Stage III indicates that the cancer has spread to both sides of the diaphragm, including one extra lymphatic organ or site.

- Stage IV shows diffuse or disseminated involvement of one or more extra lymphatic organs.

Medical Ontologies. For achieving re-usability and interoperability of data, we need an external knowledge model, that covers anatomical information related to lymphatic occurrences. Two ontologies, the Foundational Model of Anatomy (FMA) and the Radiology Lexicon (RadLex), cover anatomical entities and provide the required coverage of anatomical and radiological concepts for the staging scenario. The FMA [7] is a very comprehensive specification of anatomy taxonomy, i.e. an inheritance hierarchy of anatomical entities with different kinds of relationships. It covers approximately 70,000 distinct anatomical concepts and more than 1.5 million relations instances from 170 relation types. It provides concepts that describe single lymph nodes, such as 'axilliary_lymph_node', and concepts that describe multiple lymph nodes, such as 'set_of_axilliary_lymph_node'. It contains 425 concepts representing singular lymph nodes and 404 concepts describing sets of lymph nodes.

RadLex ${ }^{2}$ is a terminology developed and maintained by the Radiological Society of North America (RSNA) for the purpose of uniform indexing and retrieval of radiology information, including medical images. RadLex contains over 8,000 anatomic and pathologic terms, also those about imaging techniques, difficulties

\footnotetext{
${ }^{2}$ http: //www.radlex.org
} 
and diagnostic image qualities. Its purpose is to provide a standardized terminology for radiological practice. RadLex is available in English and German language and covers more than 100 concepts describing lymph node concepts.

The semantic labeling of "B-symptoms" requires an ontology that encompasses cancer-related diseases, symptoms and findings. We identified the $\mathrm{Na}$ tional Cancer Institute Thesaurus (NCI ${ }^{3}$, a standard vocabulary for cancer research, as appropriate. It contains around 34.000 concepts from which 10521 are related to Disease, Abnormality, Finding, 5901 are related to Neoplasm, 4320 to Anatomy and the rest are related to various other categories such as Gene, Protein, etc.

Semantic Image Annotation and Radiology Reports. The information about number, type and location of lymphatic occurrences can be detected by analyzing medical images. The detected information is formally captured by semantic image annotation. The MEDICO project is based on multiple ways of generating semantic image annotation. For instance methods for automated image parsing, such as [1], allow to hierarchically parse whole body CT images and efficiently segment multiple organs taking contextual information into account. While automated image parsing remains incomplete, manual image annotation remains an important complement. To integrate manual image annotation in the reporting workflow of radiologists is one of the objectives of the Annotation and Image Markup Project $([3],[2])$. Currently, users of the MEDICO system can manually add semantic image annotations by selecting or defining anatomical landmarks or arbitrary regions/volumes of interest. Ongoing work within the MEDICO project is focusing on the semi-automatically identification of terms and relations in radiology reports that are generated by clinicians in the process analyzing the patient's disease patterns by investigating medical imaging data.

\section{Knowledge-Engineering Steps for Ontology-Based Lymphoma Patient Staging}

Our goal is to integrate practical clinical knowledge into medical applications, such as decision support systems, semantic search or quality control applications. This requires the formal and explicit representation of practical clinical knowledge such that implicit conclusion can be derived. The aim of reasoning-based ontology enhancement is to use existing reasoning procedures for discovery of new classification and subsumption results.

We use OWL DL, which is MEDICO's agreed semantic representation language, for representing the knowledge model. Description Logics [8], a family of formal representation languages for ontologies, are designed for classificationbased reasoning.

Automatic symbolic reasoning deals with the formal manipulation of explicitly represented sets of symbols (defined within a formal language) using rules

3 http://nciterms.nci.nih.gov/ 
of inference. One rule of inference used is deduction where from a given set of propositions new ones can be generated and interpreted as logical consequences of these. Sets of propositions constructed in this manner and used to define a particular domain of interest are typically called a knowledge-base. The advantage of this approach is that otherwise implicit knowledge can be explicit and more readily to available to users and applications.

Definition 1 (Entailment). A knowledge base $K B$ is said to entail a statement $\alpha$ if $\alpha$ follows from the knowledge stored in the $K B$, which is written $K B$ $\models \alpha$.

The inference procedures implemented in computational reasoners aim at realizing the entailment relation between logical statements 9 , i.e. inference procedures use sets of background axioms that are implicitly used by the reasoning system. The automatic staging of lymphoma patients represented as concepts, relies on the subsumption procedure, i.e. the computing of subsumptions.

Definition 2 (Subsumption Procedure). Let $K B$ denote a knowledge base and $c, d$ be two concepts in $K B$, then we call the inference rule $\pi$ a subsumption procedure, if $\pi$ can determine for any sentence $\alpha$ of the form $(\forall x \quad x \in c \rightarrow x \in$ d) whether or not $K B \models \alpha$.

We transformed the Ann-Arbor Staging System for Hodgkin Lymphoma [5] into a formal and ontology-based representation suitable for computing subsumptions. For doing so, we follow the following steps:

\subsection{First Step: Knowledge Requirements Analysis}

For capturing the requirements of the ontology design, we followed the formal approach of [10]. We used competency questions to determine the expressiveness requirements of the knowledge model. The ontology needs to be able

1. to compute the answers to the questions using the axioms, definitions, and background axioms of the reasoning system

2. to represent the questions using its terminology

In the context of automatic classification of lymphoma patients, the Ann-Arbor Staging System specifies the scope as well as the basic constraints for developing competency questions. Each of the staging classes matches to one high-level competency questions and is represented as complex and defined OWL DL class. This provides the basis for computing the answers. i.e. the patient classification. By representing patients as OWL DL classes with the corresponding axioms and characteristics, they will be subsumed according to their staging grade.

For determining the required terminology, the decision criteria of Ann-Arbor Staging System need to be reflected. The patient classification basically relies on

- the number of lymphatic occurrences

- the relative position of a lymphatic occurrence in relation to the diaphragm 
- the type of lymphatic occurrence

- the classification along so-called "B-symptoms".

In the following step, we decomposed the complex competency questions that represent the made ontological commitment into simpler questions which we need to answer in order to answer the given questions. The simpler questions relate to the mentioned decision criteria of the Ann-Arbor Staging System.

\subsection{Second Step: Requirements Decomposition}

As already mentioned, the goal is to decompose the complex questions conforming to the Ann-Arbor Staging System for Hodgkin Lymphoma into more simple and manageable queries that represent events that can be accessed independently. For accessing the number and location of lymph node occurrences and respectively extra lymphatic organ or site involvement, as well as capture the information about possible systemic symptoms, the following queries, i.e. defined OWL classes, were specified:

- The OWL defined classes N1 and N2 subsume all patients with at least one or at least two involved lymph node regions.

- The OWL defined classes E1 and E2 subsume all patients with at least one or at least two involved extra lymphatic organ or site involvement.

- The OWL defined classes N_AllAboveD and N_AllBelowD subsume all patients with the location of the lymph node regions either only above or only below the diaphragm. For accessing patients with occurrences of lymph nodes on both sides of the diaphragm, one has to make sure that the occurrences are neither located all above nor located all below the diaphragm. This can be formulated by the complex class $\neg$ N_AllAboveD $\sqcup \neg$ N_AllBelowD.

- The location of extra nodal occurrences are identified in an analogous manner, i.e. by establishing the defined classes E_AllAboveD and E_AllBelowD, as well as the corresponding complex axiom for accessing patients with extra nodal occurrences on both sides of the diaphragm.

- The OWL defined class B-Symptom subsumes all patients with at least one systemic symptom, such as fever, night sweats or weight loss, respectively subsumes the OWL defined Class A-Symptom all patients with the absence of systemic symptoms.

Before detailing the staging classification, we will discuss the particular requirements towards the ontology design for realizing the auxiliary queries. For establishing the ontological model basically three different challenges needed to be addressed:

1. The staging of Lymphoma patients relies on many different knowledge resources, such as findings from medical image analysis findings, patient records, medical background knowledge and clinical guidelines. How to select and customize the right (fragment of) relevant knowledge resources for the integration into the staging scenario? 
2. How to ensure that the ontological model provides the basis for inferring the number of lymph node occurrences and the number of extra lymphatic involvements?

3. How to identify the relative location - above, below or on both sides of the diaphragm - of the lymphatic occurrences?

The mentioned requirements are addressed in the following steps.

\subsection{Third Step: Knowledge Integration}

The selection of the right ontologies and knowledge resources covering the domain of interest, the identification of relevant fragments or aspects of knowledge resources as basis for scalable reasoning, and the enhancement and customization of knowledge resources are important aspects for their successful integration into the intended application. The KEMM knowledge engineering methodology [6] specifies the access to the mentioned ingredients for automatic patient classification in its previous modules. We sketch them briefly in the following

Ontology Selection. As human health is a sensitive matter, the quality and the quantity of the medical knowledge to be used in the target application have to be ensured. This implies reusing the work of acknowledged resources when choosing medical ontologies. For capturing the semantics of for the staging of lymphoma patients, ontological concepts describing the organs as well as concepts listing possible regions of lymphatic occurrences are required. As the use case scenario relies only on high-level concepts, both ontologies - FMA and RadLex are suitable in terms of coverage. For this use case, we decided to use RadLex as primary third party resource to label lymphatic occurrences. As within the MEDICO project, we are confronted with radiology reports in German language, the availability of a German translation was an crucial argument for the above decision. In addition, due to the large size of Radlex, we needed to establish an ontology fragment that is scalable and efficient for reasoning application. For modeling the B-symptoms, we required concepts representing the systemic symptoms that were covered within the NCI vocabulary. As only a very small subset of NCI concepts were used within our scenario, we manually established the NCI fragment accordingly.

Ontology Modularization. Scalable reasoning requires the modularization of large ontologies. These modules need to cover all concepts and relationships for describing the particular scenario, in our case any concept describing a lymph node occurrence or an extra lymphatic involvement. Thus, we semi-automatically generated a RadLex fragment in OWL DL format following three steps:

1. For identifying the concepts representing lymph node occurrences, we selected all 'lymph node' concepts, i.e. concepts that contained the string 'lymph node' in its preferred name label. In addition, all concepts with the string 'node' in its preferred name and a 'lymph node' concept as superconcept were selected. By interviewing our clinical experts, we could derive a list of nine concepts representing extra nodal occurrences. 
2. The semantics of the parent-child relationship was translated to OWL DL axioms, such as

$$
\begin{aligned}
\text { RightLowerParaTrachealLymphnode } & \sqsubseteq \text { (Anatomical_Structure } \sqcap \\
& \sqcap \text { } \exists \text { is_child_of.LowerParaTrachealLymphnode) }
\end{aligned}
$$

3. To make sure that the the exact number of lymphatic occurrences can be inferred by the DL reasonen 4 , we needed to label the identified concepts for representing lymphatic occurrences as disjoint by adding the correlating axioms.

Ontology Customization. The relative position of lymphatic occurrences is an important decision criteria of the staging system and needs to be reflected by the reasoning procedure. The patient staging results distinguish between patient that have lymphatic occurrences only above, only below or on both sides of the diaphragm. The relative position of lymphatic occurrences to the diaphragm has to be expressed in the knowledge model. For achieving this, we see two options. The information about the relative position of lymphatic occurrences can be either derived by the image segmentation algorithm or derived directly from the underlying ontological model. In other words, this information either comes with the patient record information or with the integrated knowledge model of the human anatomy. The deduction of the relative spatial position of lymphatic occurrences by segmentation algorithms will be addressed within our future work. In the meantime, we need to rely on the selected anatomy knowledge model to capture - as basis for later inference - the relevant spatial information. FMA and RadLex do not explicitly capture the information about the relative position between lymphatic regions or organs and the diaphragm. Thus, we required to enhance the established fragment accordingly. This could be achieved by extending each lymphatic region by an axiom, such as

$$
\begin{aligned}
\text { LowerParaTrachealLymphnode } \sqsubseteq & \text { (Anatomical_Structure } \sqcap \\
& \sqcap \text { GhasN_Location.aboveDiaphragm) }
\end{aligned}
$$

indicating that the region is above, or respectively, below the diaphragm. The classification of lymphatic regions - and of patients with lymphatic occurrences above, below or on both sides of the diaphragm was modeled as value partition.

The established RadLex fragment encompasses 123 concepts, whereof 104 represent lymph node occurrences and nine extra nodal occurrences; the remaining concepts are auxiliary concepts required for the classification task.

\subsection{Fourth Step: Complex Query Composition}

The application queries are the Ann-Arbor Staging classes that are represented as defined OWL DL classes. Each staging class captures the semantics as detailed in Subsection 3. Their formal representation makes use of auxiliary classes introduced in Subsection 4.2. Figure 3 summarizes the transformation of the AnnArbor Staging System. Stage-1 is the union of the sets N1 and E1. The class N1 infers all patients with the involvement of at least one lymph node region and E1

\footnotetext{
${ }^{4}$ We use Pellet (http://clarkparsia.com/pellet/) as DL reasoner.
} 


\begin{tabular}{|l|l|}
\hline Stages & Description \\
\hline Stage-I & N1 or E1 \\
\hline Stage-II & Stage-II-N or Stage-II-mixed \\
\hline Stage-II-N & N2 and (N_AllAboveD or N_AllBelowD) \\
\hline Stage-II-mixed & N1 and E1 and ((N_AllAboveD and E_AllAboveD) or (N_AllBelowD and E_AllBelowD)) \\
\hline Stage-III & Stage-III-N or StageIII-mixed \\
\hline StageIII-N & N2 and not N_AllAboveD and not N_AllBelowD \\
\hline StageIII-mixed & E1 and N1 and (not (N_AllAboveD and E_AllAboveD) and not (N_AllBelowD and E_AllBelowD)) \\
\hline StageIV & E2 and (not N_AllAboveD and not N_AllBelowD) \\
\hline
\end{tabular}

Fig. 3. Formal Representation of Ann-Arbor Staging System

all patients with the involvement of at least one extra lymphatic organ or site. Stage-II is the union of Stage-II-N or Stage-II-mixed with Stage-II-N gathering all patients with more that two lymph node region that are all on one side of the diaphragm and Stage-II-mixed patients with one involved lymph node region and one involved extra lymphatic organ or site on the same side of the diaphragm. Patients of Stage-III are contained in Stage-III-N or in StageIII-mixed. Stage-III-N identifies all patients that have at least two involved lymph node regions that are spread to both sides of the diaphragm and StageIII-mixed all patients with one involved lymph node region and one involved extra lymphatic organ or site on both sides of the diaphragm. StageIV subsumes all patients that have two or more extra lymphatic organs or site on both sides of the diaphragm. The A-Symptom or B-Symptom classes are not indicated in the table as their semantics was already described in Sect. 4.2 .

\subsection{Evaluation}

Both, complex questions and simple questions, provided us valuable guidance for evaluating the ontological commitment that has been made. This was possible

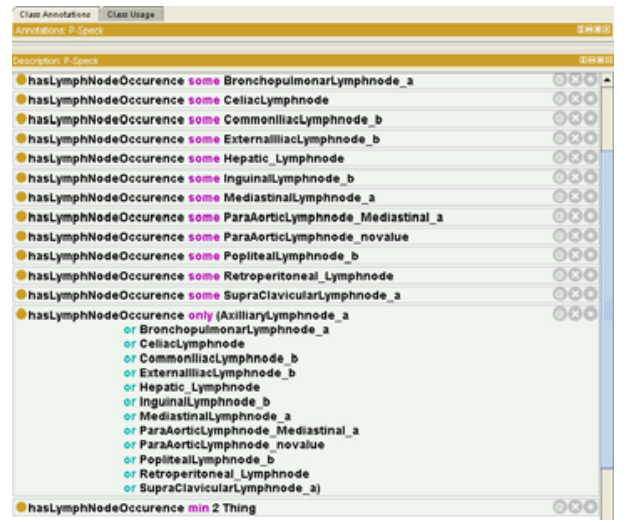

Fig. 4. OWL Representation of an example patient record 


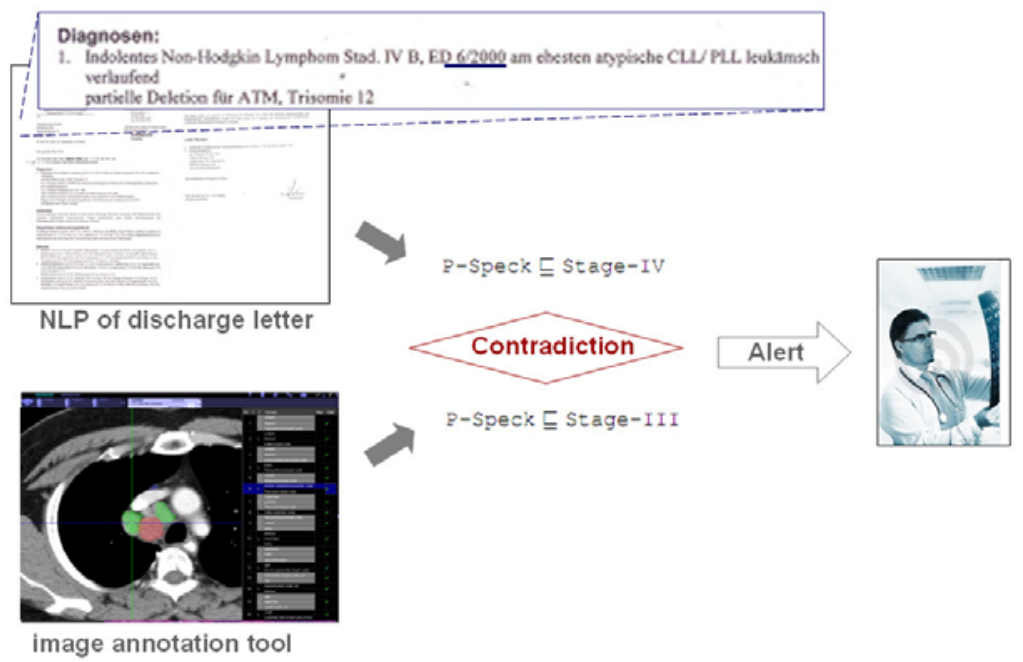

Fig. 5. Contradicting Staging Results can be discovered

by systematically establishing test patients classes according to the simple and the complex questions. Each test patient is equipped with different numbers and kinds of lymphatic occurrences at different locations in the body. The format representing the patient data is determined by the reasoning procedure. Figure 4 represents an example patient with more than 13 lymph node occurrences. By analyzing the reasoning result, we could evaluate whether the created ontology established the expressivity in accordance to the requirements of the use case scenario. In this way, the evaluation (as well as the development) of the classification axioms was straight forward. In addition, we conducted a proof-of-concept study on the basis of real patient records, which we detail in the following section.

\section{Clinical Example}

The automated patient staging approach is aiming for enhanced medical image annotation providing the basis for improved clinical applications. Thus, we conducted a proof-of-concept study on the basis of real patient records to analyze the practical potential. With the help of our clinical experts, the corresponding medical images were manually annotated and the semantic annotations integrated within our knowledge base. Additional, the information of discharge letters covering diagnose and findings was transformed into the knowledge model.

In the context of one particular patient, we identified a contradiction between the clinicians diagnose in the discharge letter, and the automated staging results based on the image annotation: The so-called Patient Speck 5 is an advanced stage lymphoma patient who was already treated with 3 chemotherapies using

5 The original name was changed. 
the CHOP-protocol. As the accomplished treatments did not help to improve the patient's health condition, he was referred to a specialist clinic.

- The patient's discharge letter covers details about the diagnose, past medical diagnose, and findings, assessment and progression and recent therapy. The discharge letter of Patient Speck indicates the diagnose of Ann-Arbor IVStage.

- In the specialist clinical, he was screened using Computer Tomography. By analyzing and annotating the medical images, 16 enlarged and pathological lymph nodes on both side of the diaphragm could be identified. But there was no indication for the involvement of extra lymphatic organs. The semantic annotations were transformed into the corresponding OWL DL representation. Relying on the formal Ann-Arbor Staging criteria, the ontology-based staging approach classifies Patient Speck as Ann-Arbor III-Stage.

The clinician's diagnose is contradicting the result of the automated reasoning application. As the staging grade strongly influences clinicians in their sequential treatment decisions, a careful handling thereof is highly recommended. By highlighting such contradicting results medical mistakes can be reduced (see Figure 51). This example case is in line with our argumentation, that the automatic generation of additional patient annotation data helps to improve medical quality control and medical care.

\section{Related Work}

\subsection{Semantic Image Understanding}

There exist a wide range of different imaging technologies and modalities, such as 4D 64-slice Computer Tomography (CT), whole-body Magnet Resonance Imaging (MRI), 4D Ultrasound, and the fusion of Positron Emission Tomography and CT (PET/CT) providing detailed insight into human anatomy, function and disease associations. Moreover, advanced techniques for analyzing imaging data generating additional quantitative parameters paving the way for improved clinical practice and diagnosis. However, for advanced applications in Clinical Decision Support and Computer Aided Diagnoses the comparative exploration of similar patient information is required. The missing link here fore is a flexible and generic image understanding. Currently, the large amounts of heterogeneous image data are stored in distributed and autonomous image databases being indexed by keywords without capturing any semantics.

Generic medical image understanding is still a long-term agenda due to the high complexity of the problem. Several challenging research questions need to be addressed for tackling this vision: For determining the scope and level of detail of the semantics of the domain, i.e. the relevant metadata for annotating medical images, one needs to find out what kind of knowledge the clinicians are interested in. The scope of the constraint domain can be determined by the set of derived query patterns [11, [12] that provide guidance in identifying the relevant 
(fragments of) ontologies [13]. Moreover, the low level features, segmentations and quantitative measures derived from image processing need to be associated with ontologies.

\subsection{Knowledge Engineering}

There has been a rapid increase in ontology building, especially within the context of the Semantic Web activities initiated by various groups from industry and academia. It has been suggested that these separate activities should be executed in a systematic manner [14, 15], as a result of which several ontology engineering methodologies have been proposed [16, 17], 18].

Some communities concentrated on developing stand-alone ontology engineering methodologies that shall be applicable across domains and tasks. Examples of these are METHONTOLOGY [18, ON-TO-KNOWLEDGE [19] and COMMON-KADS [20]. METHONTOLOGY proposes to align ontology development with software development activities and consequently defines an ontology development life cycle process (similar to software development) that consists of several phases.

Some of these methodologies are the outcome of experiences collected during the ontology development process such as the Enterprise Methodology [16] or the TOVE (Toronto Virtual Enterprise) Methodology 17. The TOVE methodology is characterized by its definition of the so-called "competency questions" that determine the scope of the ontology to be modeled and for which the future ontology shall be capable of providing answers. In developing of the ontology design for the automatic staging of lymphoma patient, we followed the approach of establishing competency questions.

\subsection{Reasoning-Based Patient Classification}

There exist several approaches that integrate practical clinical knowledge for improving clinical applications. 21] focuses on the classification of lung tumors and [22] on the classification of glioma tumors. 23] is similar to our exemplified use case, inasmuch as it introduces an application that provides support for the semantic annotation of medical images. [23] relies on the anatomy model and its regional relationships for assisting the labeling of the MRI image content by means of OWL DL reasoning. 24 aims for improved and concise patient data visualization by incorporating clinical knowledge, such as the WHO grading system. The mentioned related approaches do not provide any details about the accomplished knowledge engineering steps and we assume that the use cases could be modeled in accordance to our introduced approach.

\section{Conclusion}

The usage of OWL DL based reasoning capabilities, provide means to automatically perform lymphoma patient classification. The achieved additional patient 
annotation again can be used in a multitude of clinical applications, such as the recommendations for treatments, quality control applications, and for improved search and visualization applications for patient information. In our future work, we will focus on the integration of the established OWL DL based classification system into the overall MEDICO system aiming for improve clinical applications. Furthermore, we will analyze the potential of other reasoning approaches, such as rules or theorem proving, in the context of our application scenario.

Acknowledgments. This research has been supported in part by the THESEUS Program in the MEDICO Project, which is funded by the German Federal Ministry of Economics and Technology under the grant number 01MQ07016. The responsibility for this publication lies with the author. We are also thankful to Kamal Najib for his support with implementation tasks.

\section{References}

1. Seifert, S., Barbu, A., Zhou, S., Liu, D., Feulner, J., Huber, M., Suehling, M., Cavallaro, A., Comaniciu, D.: Hierarchical parsing and semantic navigation of full body ct data. In: SPIE Medical Imaging (2009)

2. Channin, D., Mongkolwat, P., Kleper, V., Sepukar, K., Rubin, D.: The cabib annotation and image markup project. Journal of Digital Imaging (2009)

3. Rubin, D., Mongkolwat, P., Kleper, V., Supekar, K., Channin, D.: Medical imaging on the semantic web: Annotation and image markup. In: AAAI Spring Symposium Series, Semantic Scientific Knowledge Integration, Stanford, USA (2008)

4. Möller, M., Regel, S., Sintek, M.: Radsem: Semantic annotation and retrieval for medical images. In: Aroyo, L., Traverso, P., Ciravegna, F., Cimiano, P., Heath, T., Hyvönen, E., Mizoguchi, R., Oren, E., Sabou, M., Simperl, E. (eds.) ESWC 2009. LNCS, vol. 5554, pp. 21-35. Springer, Heidelberg (2009)

5. Wittekind, C., Meyer, H., Bootz, F.: TNM Klassifikation maligner Tumoren. Springer, Heidelberg (2005)

6. Wennerberg, P., Zillner, S., Möller, M., Buitelaar, P., Sintek, M.: Kemm: A knowledge engineering methodology in the medical domain. In: Proc. of the 5th International Conference on Formal Ontology in Information Systems, FOIS (2008)

7. Rosse, C., Mejino, J.J.: A reference ontology for biomedical informatics: the foundational model of anatomy. J. of Biomedical Informatics 36 (2003)

8. Baader, F., Calvanese, D., McGuinness, D.L., Nardi, D., Patel-Schneider, P.F. (eds.): The Description Logic Handbook: Theory, Implementation, and Applications. Cambridge University Press, Cambridge (2003)

9. Russel, S., Norvig, P.: Artificial Intelligence: A Modern Approach. Prentice Hall, New Jersey (1995)

10. Uschold, M., Gruninger, M.: Ontologies: Principles, methods and applications. Knowledge Engineering Review 11 (1996)

11. Wennerberg, P., Buitelaar, P., Zillner, S.: Towards a human anatomy data set for query pattern mining based on wikipedia and domain semantic resources. In: Proceedings of a Workshop on Building and Evaluating Resources for Biomedical Text Mining (LREC), Marrakech, Marocco (2008)

12. Buitelaar, P., Wennerberg, P., Zillner, S.: Statistical term profiling for query pattern mining. In: Proceedings of ACL 2008 BioNLP Workshop, Columbus, Ohio, USA (2008) 
13. Wennerberg, P., Zillner, S.: Towards context driven modularization of large biomedical ontologies. In: Proceedings of the International Conference of Biomedical Ontology (ICBO), Buffalo, New York, US (2009)

14. Bouaud, J., Bachimont, B., Charlet, J., Zweigenbaum, P.: Methodological principles for structuring an "ontology" (1995)

15. Jones, D., Bench-Capon, T., Visser, P.: Methodologies for ontology development (1998)

16. Uschold, M.: Building ontologies: Towards a unified methodology. In: 16th Annual Conf. of the British Computer Society Specialist Group on Expert Systems (1996)

17. Gruninger, M., Fox, M.S.: The design and evaluation of ontologies for enterprise engineering (1994)

18. López, M.F., Gómez-Pérez, A., Sierra, J.P., Sierra, A.P.: Building a chemical ontology using methontology and the ontology design environment. IEEE Intelligent Systems 14 (1999)

19. Sure, Y., Studer, R.: On-to-knowledge methodology - final version (2002)

20. Schreiber, G., Akkermans, H., Anjewierden, A., Dehoog, R., Shadbolt, N., Vandevelde, W., Wielinga, B.: Knowledge Engineering and Management: The CommonKADS Methodology. The MIT Press, Cambridge (1999)

21. Dameron, O., Roques, E., Rubin, D., Marquet, G., Burgun, A.: Grading lung tumors using owl-dl based reasoning. In: Proceedings of 9th International protégé Conference (2006)

22. Marquet, G., Dameron, O., Saikali, S., Mosser, J., Burgun, A.: Grading glioma tumors using owl-dl and nci-thesaurus. In: Proceedings of the American Medical Informatics Association Conference AMIA (2007)

23. Golbreich, C., Dameron, O., Bierlaire, O., Gibaud, B.: What reasoning support for ontology and rules? the brain anatomy case study. In: Proceedings of the Workshop on OWL Experiences and Directions, Irlande (2005)

24. Zillner, S., Hauer, T., Rogulin, D., Tsymbal, A., Huber, M., Solomonides, T.: Semantic visualization of patient information. In: Proceedings of the 21th IEEE International Symposium on Computer-Based Medical Systems (CBMS), Jyvskyl, Finland (2008) 